\title{
Zona planto-pédieux révélateur de l'infection à VIH.
}

\section{Planto-Pedal Zona indicative of HIV infection.}

\author{
Keita $\mathbf{L}^{1}$, Fofana $\mathrm{Y}^{1}$, Sissoko $\mathrm{M}^{3}$, Traore $\mathrm{B}^{1}$, Diaby $\mathrm{MG}^{2}$, Cisse $\mathrm{L}^{4}$, Karabinta $\mathrm{Y}^{2}$, Dicko AA ${ }^{1,2}$, Faye $\mathrm{O}^{1,2}$ \\ 1-CNAM,Ex_Institut Marchoux à Djicoroni-Para \\ 2- FMOS \\ 3-Hôpital Régional de Gao \\ 4-Centre de Santé de Référence de Koulikoro \\ Auteur correspondant : Dr_Lassine Keïta, CNAM Bamako Email : 1assinekeita70@yahoo.fr
}

\section{Résumé :}

Le zona est une dermatose virale qui peut intéresser n'importe quelle partie du corps. La plus part des personnes atteintes d'un zona ont entre 50 à 70 ans. Toutefois son apparition chez le sujet jeune doit fait rechercher une immunodépression à VIH. Nous rapportons un cas atypique de zona révélant l'infection à VIH chez une patiente de 25 ans présentant une éruption vésiculeuse, douloureuse reposant sur un fond érythémateux localisé sur la plante du pied gauche. Le diagnostic du zona a été évoqué grâce aux éléments cliniques et réconforté par le cytodiagnostic et l'histologie. Cette localisation atypique nous a incités à demander une sérologie à VIH qui est revenue positive.

Mots-clés : zona, atypique, VIH

Abstract :

Shingles is a viral dermatosis that can affect any part of the body.Most people with shingles are between 50 and 70 years old. However, its appearance in young people must seek immunosuppression to HIV. We report an atypical case of shingles revealing HIV infection in a 25 -year-old patient with a painful vesicular eruption on an erythematous fundus located on the sole of her left foot. The diagnosis of shingles has been discussed with clinical evidence and comforted by cytodiagnosis and histology. This atypical localization prompted us to seek positive HIV serology.

Keywords: shingles, atypical, HIV

Introduction: Le zona est une infection cutanée causée par la réactivation du virus zona-varicelle (VZV). Il est caractérisé par des lésions vésiculaires groupées qui suivent la distribution d'un dermatome, le plus souvent unilatéral, et accompagnées de douleur [1]. Son incidence augmente après 50 ans pour atteindre son maximum au-delà de 75 ans. Le zona est considéré actuellement comme un signe d'orientation clinique sûr avec des valeurs prédictives positives qui varient de $80-100 \%$ [2]. Les sujets immunodéprimés sont particulièrement exposés à développer un zona, notamment les patients traités par corticothérapie prolongée ou chimiothérapie anticancéreuse, ceux transplantés, les individus infectés par le virus de l'immunodéficience humaine, ou les porteurs d'atteintes malignes hématologiques [3]. Sa survenue chez l'adulte jeune doit faire rechercher une infection à VIH [4]. Nous rapportons un cas atypique de zona révélant l'infection à VIH.

Observation: Il s'agissait d'une demoiselle de 25 ans aux antécédents de varicelle dans l'enfance, vue en consultation dermatologique pour une éruption plantaire apparue 48 heures plus tôt, marquée par une douleur insomniaque à type de constriction, de sensation de brûlure et parfois une hyperesthésie importante rendant la marche difficile. L'examen clinique retrouvait une éruption siégeant sur l'hémiface plantaire interne du pied gauche sur un fond érythémateux, surmonté de vésicules enchâssées sous la peau et groupées en bouquet par endroit sans déborder sur la hémiface controlatérale. Puis la progression se faisant sur la jambe en suivant un trajet métamérique. Le bilan de laboratoire a révélé une sérologie rétrovirale positive à VIH, le cytodiagnostic de Tzank a mis en évidence une altération des kératinocytes traduisant une dégénérescence ballonisante de Unna. Et l'histologie montre des vésicules intra-épidermiques avec des inclusions intranucléaires et un infiltrat leucocytaire polymorphe présent dans le derme. Un traitement fut institué : un Antiseptique solution, Paracétamol $325 \mathrm{mg}+$ Chlorydrate de Tramadol 37, $50 \mathrm{mg}$, Acyclovir $200 \mathrm{mg}$ et Amitriptyline 4\% goutte. Au bout de 72 heures la douleur a considérablement diminué et la patiente pouvait reprendre ses activités quotidiennes. 


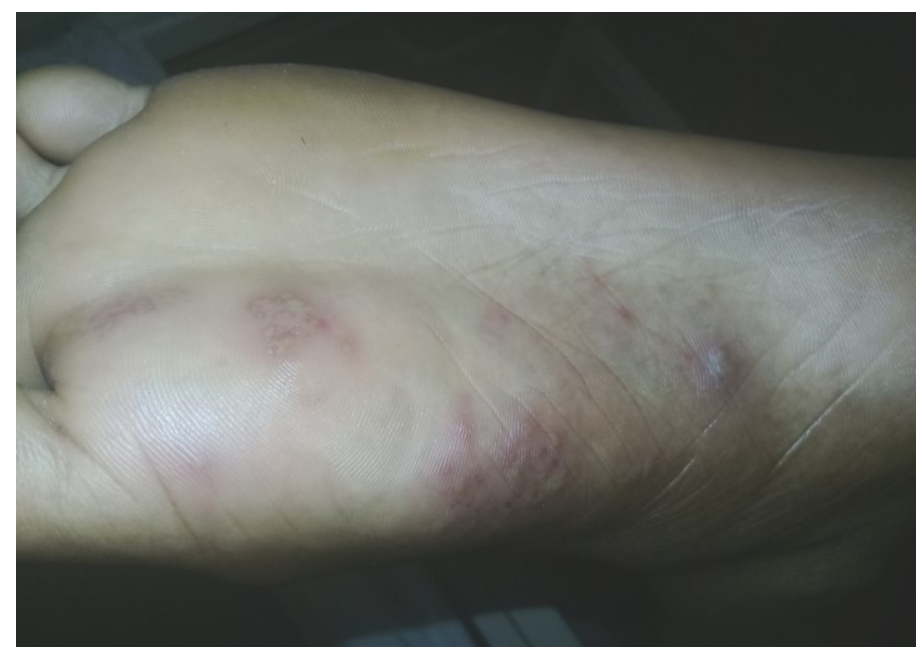

Fig 1: Avant traitement ( plante du pied)

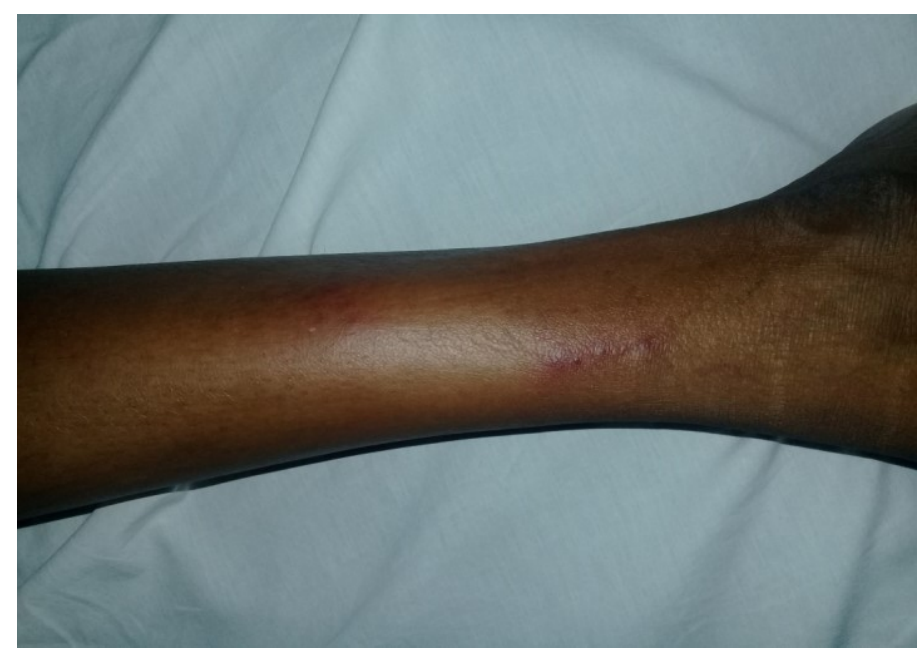

Fig 2 : Avant traitement (Jambe)

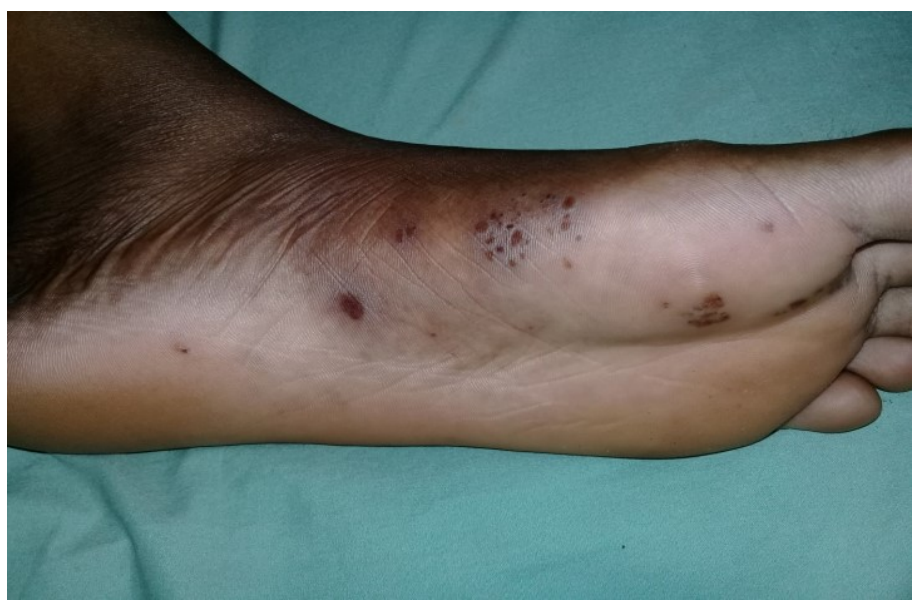

Fig 3 : Après traitement ( 3 jours)

Discussion : Les sujets présentant un déficit de l'immunité cellulaire sont susceptibles de développer des formes plus sévères ou atypiques du zona [5]. C'est une manifestation très fréquente de la séropositivité VIH pouvant survenir à tous les stades, mais souvent contemporaine d'une immunodépression déjà grave [6]. Ces lésions peuvent être uniques ou multiples et se situer à n'importe quel endroit du tégument. Elles évoluent sur plusieurs 
semaines à plusieurs mois alternant des phases d'extension et de régression sans guérison complète [7]. Des différentes topographies des lésions de zona ont été rapportées dans la littérature [8], mais une localisation planto-pédieuse n'a fait l'objet d'aucune publication à notre connaissance. Cette localisation prête à confusion avec d'autres dermatoses vésiculeuses notamment la dyshidrose, une forme particulière d'eczéma dans laquelle les vésicules siègent aux faces latérales des doigts et des orteils ainsi qu'aux paumes des mains et aux plantes des pieds [9]. Cette éruption indolore se caractérise de vésicules dures, tendues, enchâssées dans l'épiderme, qui sont souvent groupées en placards de forme arrondie, polycyclique ou serpigineuse sur une base érythémateuse. Mais l'éruption du zona est douloureuse avec des accès paroxystiques, accompagnée de paresthésies et de troubles objectifs de la sensibilité [10]. Le diagnostic peut être aussi confondu avec l'herpès cutané qui se caractérise par des vésicules intra-épidermiques ayant tendance à se regrouper en bouquet, à contour polycyclique, ubiquitaire, il intéresse en particulier le doigt, la joue, le tronc [11], et la localisation plantaire, ainsi que les signes généraux sont rares.

\section{Conclusion}

Le zona est une pathologie bénigne qui apparaissait surtout fréquent chez les âgés (50-70 ans), mais sa survenue chez l'adulte jeune nous a motivé à rechercher une immunodépression à VIH devant sa présentation clinique inhabituelle.

\section{Conflit d'intérêt : aucun}

\section{Références}

1-Dombrey H.Herpes zoster in infants.Folia Med (Plovdiv). 1994 ; 36 (4) 45-49.

2-Tolaifeht, Zainab A., ALHATTAB, Mohammed K., et NAHER, Habeeb S. The role of cell mediated immunity in reactivation of latent Varicella-Zoster virus. International Journal of Chem. Tech. Research.2017 ; $10(2): 655-661$.

3- RUBIN, Lorry G, LEVIN, Myron J., LJUNGMAN, Per, et al. 2013 IDSA clinical practice guideline for vaccination of the immunocompromised host. Clinical Infectious Diseases. 2013; 58 (3) :44-100

4- CHEN, Guan-Jhou, LIN, Kuan-Yin, SUN, Hsin-Yun, et al. Incidence of acute hepatitis A among HIV-positive patients during an outbreak among MSM in Taiwan: Impact of HAV vaccination. Liver International.2018;38 (4) : 594-601.

5- Geretti, A. M., Brook, G., Cameron, C., Chadwick, D., French, N., Heyderman, R. ... \& Mac Mahon, E. (2016). B ritish HIV A ssociation Guidelines on the Use of Vaccines in HIV-Positive Adults.HIV medicine.2015; 17: 2-81

6-Buchbinder SP, Katz MH, Hessol NA, Liu JY, O'Malley PM, Underwood R, et al. Herpes zoster and human immunodeficiency virus infection. J Infect Dis. 1992; 166(5):1153-6

7- Gershon AA, Mervish N, Larussa P, Steinberg S, Lo SH,Hodes D et al. Varicella zoster virus infection in children with underlying human immunodeficiency virus infection. J infect Dis 1997; 176: 1496-1500

8-Saurat J H,Lachapelle JM,Lipsker D,Thomas L. Viroses à expression cutanée.Dermatologie et Infections sexuellement transimissibles.Elsevier/Masson.2015; 99-136

9- Lachapelle JM, Tennsted D. La dyshidrose et l'eczéma dyshidrosique : approches diagnostiques et thérapeutiques innovatrices. Louvain Med.2000; 119 :S55 -S63.

10-Njamnshi AK, Bissek AC, Djientcheu V de P, Mbuagbaw J, Yepnjio FN, Ongolozogo P, Njock R, Luma HN, Ngwane S, Lando MJ, Mbua B,Kaptue L N,Muna W FT. Le zona et l'infection à VIH au Cameroun: Aspects épidémiologiques et cliniques.Health sciences and disease.2000;3:5-21

11-Onunu AN, Uhunmwangho A. Clinical spectrum of herpes zoster in HIV-infected versus non HIV infected patients Benin City, Nigeria. West Afr J Med.2004;23(4):300-4 